\title{
Valutazione metabolica della nefrolitiasi recidivante
}

\author{
M. Lombardi ${ }^{1}$, S. Michelassi ${ }^{1}$, R. Santoni ${ }^{2}$, S. Dolenti ${ }^{3}$, A. Amodei ${ }^{4}$ \\ ${ }^{1}$ Unità Operativa di Nefrologia e Dialisi, Ospedale S.M. Annunziata, Antella, Firenze \\ ${ }^{2}$ Laboratorio di Analisi, Ospedale S.M. Annunziata, Antella, Firenze \\ ${ }^{3}$ Laboratorio di Analisi, Ospedale IOT, Firenze \\ ${ }^{4}$ Unità Operativa di Dietetica, Ospedale S.M. Annunziata, Antella, Firenze
}

$\mathbf{N}$

egli anni '90, dopo l'introduzione delle moderne tecniche endourologiche e della litotripsia extracorporea, si è assistito ad una rivoluzione nel trattamento della nefrolitiasi $(1,2)$. La maggior parte dei calcoli urinari possono ora essere rimossi con maggior facilità e minor morbilità.

Il miglioramento di tali metodiche, associato al momento di recessione in atto nel mondo della sanità, hanno generato l'erronea tendenza di ritenere superflue, superate ed onerose quelle indagini $m e$ diche che portano ad una diagnosi eziopatogenetica (3).

Al contrario, per ottenere il controllo della nefrolitiasi recidivante, non si può prescindere dall'approccio medico: in primo luogo perché l'eliminazione dei calcoli, anche se più facilmente raggiungibile, non previene la recidiva; in secondo luogo perché solo evidenziando la presenza di errori metabolici e correlandoli agli eccessi dietetici si può porre una diagnosi, prescrivere una terapia medico-dietetica e formulare una prognosi.

\section{Epidemiologia della nefrolitiasi}

Nei Paesi industrializzati la nefrolitiasi ha una prevalenza che varia dall' 1 al $5 \%$, con un'incidenza annuale dello 0.1-
$0.3 \%$. La maggior parte dei calcoli (75\%) sono composti da ossalato di calcio $(\mathrm{OXCa})$ o fosfato di calcio $(\mathrm{PCa})$; il restante $25 \%$ sono composti da acido urico, cistina o magnesio-ammonio-fosfato (struvite). L'elevato impatto sociale della nefrolitiasi, specialmente quella calcica, è dovuto alla sua elevata prevalenza (dal 4 al 10\% della popolazione generale adulta) e dalla sua frequenza di recidiva $(70-80 \%)$ (4). La maggior suscettibilità per la calcolosi di $\mathrm{OXCa}$, si ha nel sesso maschile durante il middleaged period, mentre la calcolosi di PCa e struvite predominano nel sesso femminile. Conoscere la composizione fisicochimica dei calcoli può talvolta facilitare l'orientamento diagnostico, come nel caso dei calcoli di cistina, di acido urico, di struvite e di PCa (Tab. I).

\section{Classificazione eziopatogenetica della nefrolitiasi}

Le nostre conoscenze sulla eziopatogenesi della nefrolitiasi si sono progressivamente ampliate nel corso degli ultimi 20 anni. Oggi sappiamo che i pazienti affetti da nefrolitiasi possono essere portatori di una grande varietà di disturbi fisiologici, ritenuti patogeneticamente importanti nella formazione dei calcoli renali (5). È ormai universalmente accettato che queste alterazioni fisiopatologiche sono causate sia da fattori ambientali (abitudini alimentari, attività lavorative e ricreative, latitudine geografica, ecc.), che da fattori metabolici (errori genetici,

TAB. I - IMPORTANZA DELL'ANALISI DEI CALCOLI NELL'ORIENTAMENTO DIAGNOSTICO

\section{Composizione fisico-chimica}

Cistina

Acido urico

Struvite

$\mathrm{PCa}$
Orientamento diagnostico

Cistinuria

Diatesi uratica

Calcolosi infetta

Acidosi tubulare renale distale,

Iperparatiroidismo primitivo

OXCa (ac. urico)
Ampia varietà di errori metabolici e dietetici 
TAB. II - CLASSIFICAZIONE DELLA NEFROLITIASI

\section{NEFROLITIASI CALCICA}

Da ipercalciuria

Assorbitiva

Tipo I
Tipo II
Tipo III

Renale

Riassorbitiva

Da iperuricosuria

Dietetica

Eccessiva produzione endogena di acido urico

Da iperossaluria

Primitiva

Secondaria

Dietetica

Malattie intestinali

Da deficienza di inibitori urinari

Citrato (Ipocitraturie)

Altri (magnesio, GAGS, glicoproteine, ecc.)

Da basso volume urinario

Senza evidenti alterazioni metaboliche

\section{NEFROLITIASI NON CALCICA}

Diatesi gottosa (calcolosi da acido urico)

Cistinuria (calcolosi da cistina)

Calcolosi infetta con germi urea-splitting (calcoli di struvite)

(GAGS=glicosaminoglicani)

ormonali, ecc). Ha preso corpo infatti negli anni '90 una teoria, definita 'Powder Keg and Tinderbox Theory' (6), che vede la compartecipazione di entrambi i fattori nella genesi della maggior parte dei casi di nefrolitiasi.

L'analisi delle alterazioni metaboliche ed ambientali presenti nei pazienti nefrolitiasici ha portato alla attuale complessa classificazione della nefrolitiasi (Tab. II) (7). La sua importanza deriva dal fatto che essa ha permesso un più razionale approccio al trattamento medico della calcolosi renale (8).

Con un'adeguata valutazione anamnestica, dietetica, metabolica e con l'indispensabile ausilio radiologico è possibile ricondurre oltre il $95 \%$ dei casi ad una delle forme riportate nella seguente classificazione (Tab. II) (9).
Deve essere sottolineato che spesso le forme eziopatogenetiche di seguito descritte possono associarsi tra loro.

\section{NEFROLITIASI CALCICA}

\section{Nefrolitiasi da ipercalciuria}

Si definisce ipercalciuria la presenza di un'elevata escrezione urinaria di calcio, superiore a 250 e $300 \mathrm{mg} /$ die rispettivamente nel sesso femminile e maschile (10). L'ipercalciuria può provocare nefrolitiasi incrementando la saturazione urina- ria dei sali costituenti i calcoli (OXCa, $\mathrm{PCa}$, ad esempio) e/o riducendo l'attività degli inibitori urinari (il calcio urinario si può legare, ad esempio, alle cariche negative di inibitori quali citrato, GAGS, ecc.) (11).

La calcolosi da ipercalciuria comprende numerose entità di origine eterogenea. Quella di più frequente riscontro è causata da un aumentato assorbimento intestinale di calcio, indipendente dai livelli di vitamina D circolante, definita Ipercalciuria Assorbitiva di tipo I e II (12). La causa esatta dell'iper-assorbimento intestinale non è nota; potrebbe essere dovuta ad un difetto primitivo - a carattere ereditario autosomico dominante - dell'assorbimento del calcio in una parte dell'intestino (Digiuno) $(13,14)$. L'iper-assorbimento intestinale di calcio, ne aumenta i livelli plasmatici, incrementando il suo carico filtrato dal rene e inibendo contemporaneamente la funzione paratiroidea. I ridotti livelli di paratormone circolante (PTH) sono a loro volta responsabili della diminuzione del riassorbimento tubulare distale di calcio, causa dell'eccessiva perdita renale di calcio (ipercalciuria). Quest'ultima, compensando l'eccessivo assorbimento intestinale di calcio, mantiene la calcemia entro normali valori con una funzione paratiroidea modestamente soppressa (Fig. 1).

L'ipercalciuria può essere sostenuta, in una minor parte dei casi, da un primitivo disordine renale, nel quale il tubulo distale è incapace di riassorbire adeguatamente il calcio filtrato a livello glomerulare: Ipercalciuria Renale (Fig. 2) (12). Al contrario delle forme assorbitive, la perdita renale di calcio provoca una riduzione della concentrazione del calcio plasmatico con secondario stimolo della funzione paratiroidea. Ciò comporta un aumento della mobilizzazione ossea del calcio (> riassorbimento osseo) e dell' assorbimento intestinale dello ione per incremento della sintesi renale di vitamina $1,25-(\mathrm{OH})_{2} \mathrm{D}$. Classicamente in questa forma, i livelli di calcio circolante restano nei limiti della norma nonostante la perdita renale di calcio proprio grazie all'instaurarsi di un lieve iperparatiroidismo secondario (Fig. 2). L'Ipercalciuria Riassorbitiva è quella forma di ipercalciuria dovuta ad iperparatiroidismo primitivo, descritta in Figura 3. Un aumentato riassorbimento osseo è comunque presente anche in altri stati patologici, che si differenziano dal classico iperPTH primitivo proprio per il mancato 


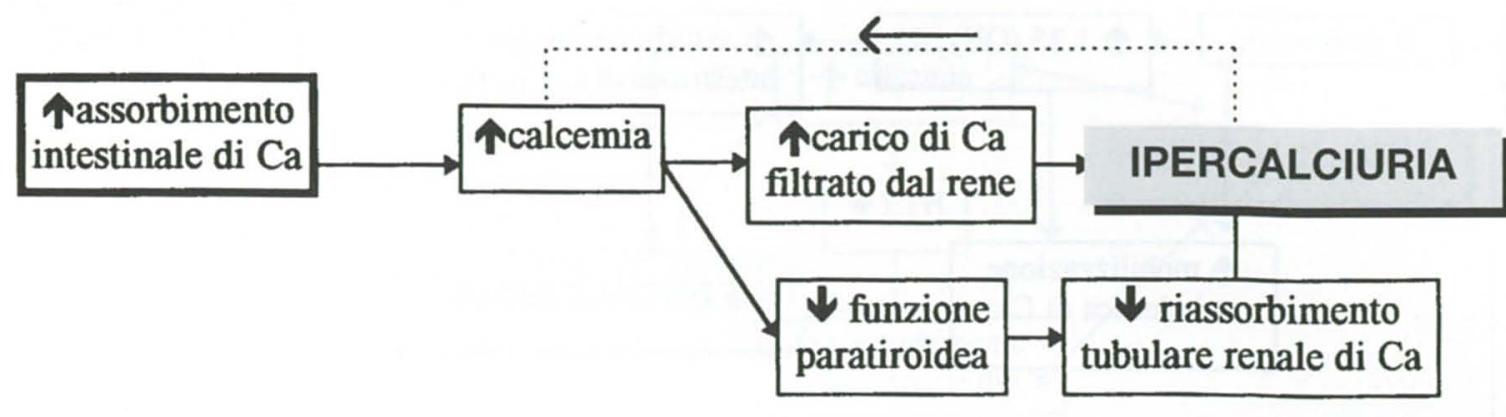

Fig. 1 - Schema patogenetico dell'Ipercalciuria Assorbitiva tipo I e II.

aumento della funzione paratiroidea, che anzi tende ad essere soppressa dalla mobilizzazione ossea di calcio. Tra i principali quadri di ipercalciuria da riassorbimento secondario sono da annoverare quello da prolungata immobilizzazione, da osteolisi in corso di neoplasie primitive o ripetitive e da ipertiroidismo (9). Una componente di riassorbimento osseo si ha anche in corso di sarcoidosi, terapie con steroidi e nelle acidosi tubulari renali (RTA) (9).

È poi descritto un quadro clinico di ipercalciuria primitiva a cavallo tra le forme Assorbitive e Riassorbitive. Si tratta di quella forma di assai raro riscontro, definita Ipercalciuria Assorbitiva tipo III, secondaria ad una tubulopatia fosfodisperdente che genera ipofosforemia (15). Quest'ultima provoca un incremento della sintesi di vitamina D che a sua volta porta ad aumento dell'assorbimento intestinale di calcio e della sua mobilizzazione ossea, già stimolata di per sé dall'ipofosforemia (Fig. 4).

Infine in circa il $18 \%$ dei casi di calcolosi da ipercalciuria, il riassorbimento osseo viene attribuito a cause varie e di difficile approccio diagnostico per un normale laboratorio di analisi (Tab. III) (6).

Da questi principali momenti patogenetici delle Ipercalciurie derivano quei criteri diagnostici, oramai codificati da tempo, grazie ai quali si può porre in atto il corretto inquadramento eziopatologico delle ipercalciurie. Tali criteri diagnostici sono riassunti nella Tabella IV.

Eppure ancora oggi, in una buona parte dei casi, il paziente affetto da calcolosi renale non riesce ad ottenere la caratterizzazione del sottotipo di ipercalciuria che lo affligge (16), neppure la generica diagnosi di 'Ipercalciuria Idiopatica', dizione coniata nel 1958 (17) e caduta in proscrizione da oltre vent'anni.

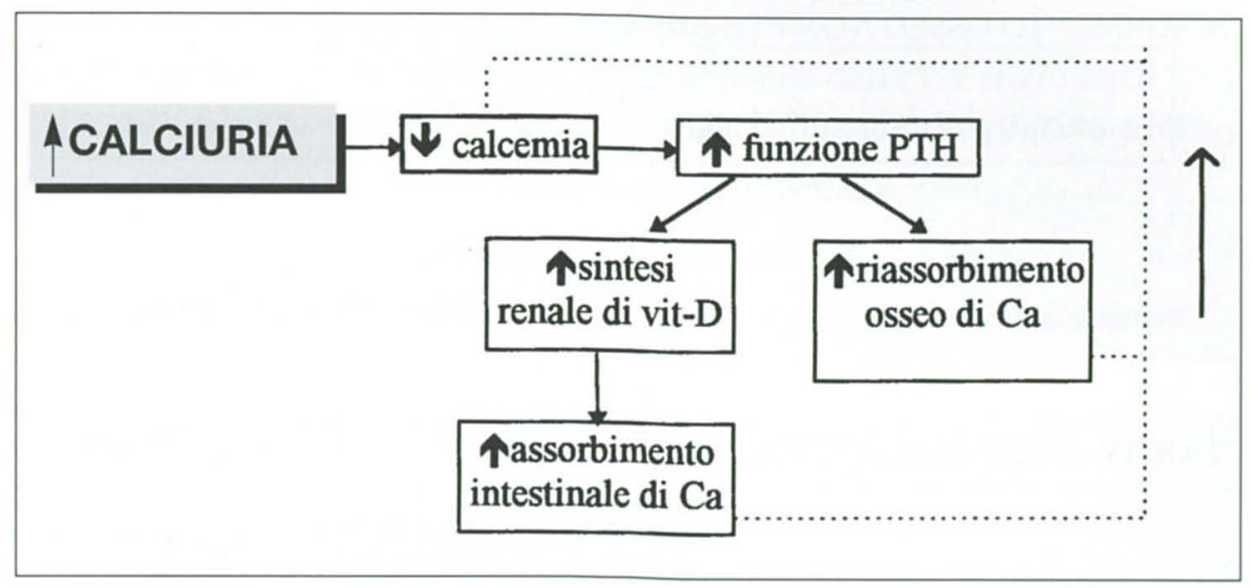

Fig. 2 - Schema patogenetico dell'Ipercalciuria Renale.

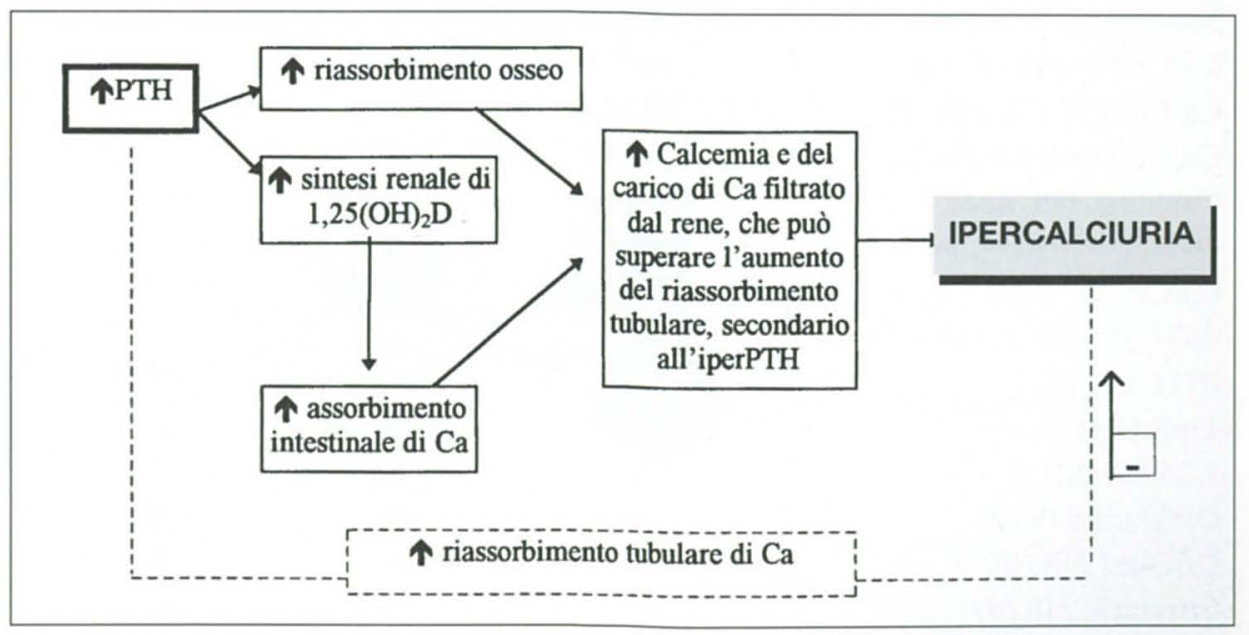

Fig. 3 - Schema patogenetico dell'Ipercalciuria Riassorbitiva da iperparatiroidismo primitivo.

\section{Nefrolitiasi (calcica) da iperuricosuria}

Un appropriato studio metabolico è in grado di identificare, tra i pazienti affetti da nefrolitiasi calcica recidivante, un $7 \%$ circa in cui è evidenziabile il solo dato di iperuricosuria in presenza di un $\mathrm{pH}$ urinario > 5.5 (Tab. II e VIII) (9). La diagnosi di questa forma è più difficile di quanto possa sembrare perché essa si associa spesso alle forme di ipercalciuria sopramenzionate (20\%) (9). 


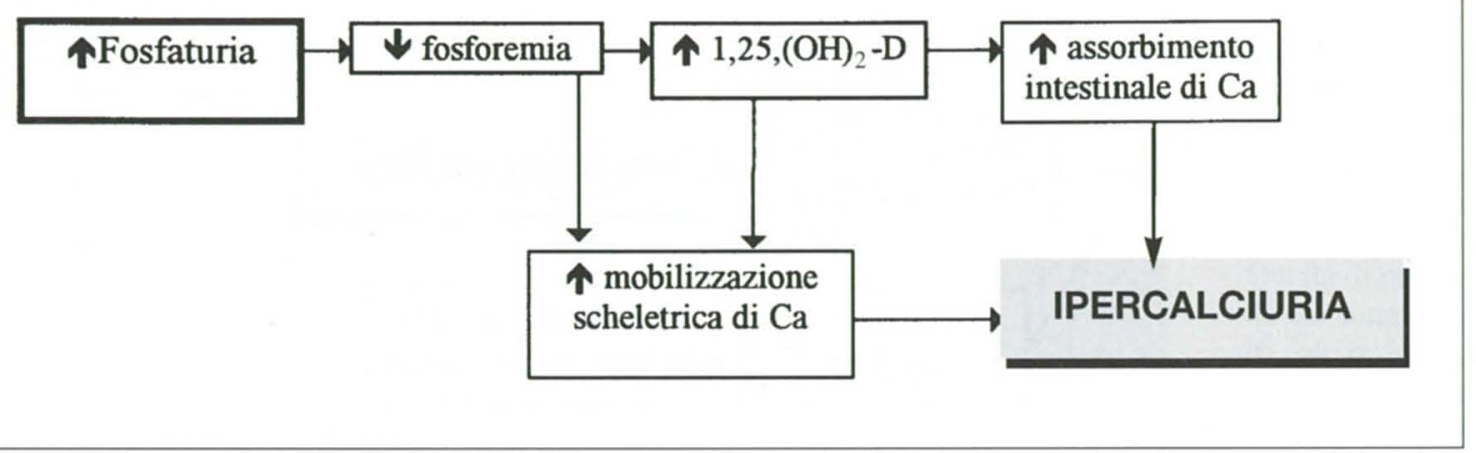

Fig. 4 - Schema patogenetico dell'Ipercalciuria Assorbitiva tipo III.

TAB. III - POSSIBILI CAUSE DI IPERCALCIURIA DA AUMENTATO RIASSORBIMENTO OSSEO NON-PTH-MEDIATO

Primitiva iperproduzione di $1,25,(\mathrm{OH})_{2} \mathrm{D}$ (senza perdita renale di fosfati) (Fig. 5) Aumento dei recettori ossei della Vit-D

Eccesso di prostaglandine

Aumentata produzione di citokine (IL-1) da parte dei macrofagi del midollo osseo
L'ambiente urinario di tali pazienti è sovrasaturo di urato monosodico e/o acido urico (con prevalenza dell'uno o dell'altro in relazione al $\mathrm{pH}$ urinario). Questi due prodotti del metabolismo purinico, la cui origine è schematizzata in Figura 6 , possono iniziare la formazione di un calcolo calcico per nucleazione eteroge$n e a$, ovvero fornendo il nucleo di partenza che permette la successiva cristallizzazione dell'OXCa, o per crescita epi-

TAB. IV - PRINCIPALI CRITERI DIAGNOSTICI DELLE IPERCALCIURIE

\begin{tabular}{|c|c|c|c|c|c|c|}
\hline & $\begin{array}{l}\text { Assorbitiva } \\
\text { tipo I }\end{array}$ & $\begin{array}{l}\text { Assorbitiva } \\
\text { tipo II }\end{array}$ & Renale & $\begin{array}{l}\text { IperPTH } \\
\text { primitivo }\end{array}$ & $\begin{array}{l}\text { Assorbitiva } \\
\text { tipo III }\end{array}$ & Riassorbitiva \\
\hline Calcemia & $\mathrm{N}$ & $\mathrm{N}$ & $\mathrm{N}$ & $\uparrow$ & $\mathrm{N}$ & $\mathrm{N}$ \\
\hline Fosforemia & $\mathrm{N}$ & $\mathrm{N}$ & $\mathrm{N}$ & $\downarrow$ & $\downarrow$ & $\mathrm{N}$ \\
\hline $\mathrm{Ca} / \mathrm{Cr} 24 \mathrm{~h}$ dieta libera & $>0.2$ & $>0.2$ & $>0.2$ & $>0.2$ & $>0.2$ & $>0.2$ \\
\hline $\mathrm{Ca} / \mathrm{Cr} / \mathrm{GFR}$ a digiuno $\left({ }^{\circ}\right)$ & $<0.11$ & $<0.11$ & $>0.11$ & $>0.11$ & $>0.11$ & $>0.11$ \\
\hline $\mathrm{Ca} / \mathrm{Cr}$ dopo carico di $\mathrm{Ca}$ & $>0.2$ & $>0.2$ & $>0.2$ & $>0.2$ & $>0.2$ & $>0.2$ \\
\hline $\begin{array}{l}\text { Calciuria } 24 \mathrm{~h} \text {, dopo } 7 \mathrm{gg} \\
\text { dieta ipocalcica e iposodica }\end{array}$ & $\begin{array}{l}>200 \\
\mathrm{mg} / \mathrm{die}\end{array}$ & $\begin{array}{l}<200 \\
\mathrm{mg} / \mathrm{die}\end{array}$ & $\begin{array}{l}>200 \\
\mathrm{mg} / \text { die }\end{array}$ & $\begin{array}{l}>200 \\
\mathrm{mg} / \mathrm{die}\end{array}$ & $\begin{array}{l}>200 \\
\mathrm{mg} / \mathrm{die}\end{array}$ & $\begin{array}{l}>200 \\
\mathrm{mg} / \mathrm{die}\end{array}$ \\
\hline $\begin{array}{l}\mathrm{Ca} / \mathrm{Cr} 24 \mathrm{~h} \text {, dopo } 7 \mathrm{gg} \mathrm{di} \\
\text { dieta ipocalcica e iposodica }\end{array}$ & $>0.2$ & $<0.2$ & $>0.2$ & $>0.2$ & $>0.2$ & $>0.2$ \\
\hline PTH-s & $\mathrm{N} \downarrow$ & $\mathrm{N} \downarrow$ & $\uparrow$ & $\uparrow \uparrow$ & $\mathrm{N}$ & $\mathrm{N} \downarrow$ \\
\hline TmP/GFR & $\mathrm{N}$ & $\mathrm{N}$ & $\mathrm{N}$ & $\mathrm{N} \downarrow$ & $\downarrow$ & $\mathrm{N}$ \\
\hline $1,25,(\mathrm{OH})_{2} \mathrm{D}$ & $\mathrm{N}$ & $\mathrm{N}$ & $\uparrow$ & $\uparrow$ & $\uparrow$ & $\mathrm{N}$ \\
\hline Ossaluria $24 \mathrm{~h}(\#)$ & $\mathrm{N}$ & $\mathrm{N}$ & $\mathrm{N}$ & $\mathrm{N}$ & $\mathrm{N}$ & $\mathrm{N}$ \\
\hline Uricuria 24h (\#) & $\mathrm{N}$ & $\mathrm{N}$ & $\mathrm{N}$ & $\mathrm{N}$ & $\mathrm{N}$ & $\mathrm{N}$ \\
\hline Citraturia 24h (\#) & $\mathrm{N}$ & $\mathrm{N}$ & $\mathrm{N}$ & $\mathrm{N}$ & $\mathrm{N}$ & $\mathrm{N}$ \\
\hline $\mathrm{pH}-\mathrm{u} 24 \mathrm{~h}$ & $\mathrm{~N}$ & $\mathrm{~N}$ & $\mathrm{~N}$ & $\mathrm{~N}$ & $\mathrm{~N}$ & $\mathrm{~N}$ \\
\hline Marker di osteolisi $(*)$ & $?$ & $?$ & $?$ & $\uparrow$ & $?$ & $\mathrm{~N} \uparrow$ \\
\hline Marker di osteogenesi $(\S)$ & $?$ & $?$ & ? & $\uparrow$ & $?$ & $?$ \\
\hline Densitometria ossea & $\mathrm{N} \downarrow$ & $\mathrm{N} \downarrow$ & $\downarrow$ & $\downarrow \downarrow$ & $\downarrow$ & $\downarrow$ \\
\hline
\end{tabular}

Legenda: $\mathrm{N}=$ normale; $\uparrow=$ aumento; $\downarrow=$ diminuzione; $\mathrm{Ca} / \mathrm{Cr} / \mathrm{GFR}=$ calciuria/creatininuria per $100 \mathrm{ml}$ filtrato glomerulare; $\mathrm{TmP} / \mathrm{GFR}=\mathrm{sog}$ lia renale dei fosfati; $\mathrm{pH}-\mathrm{u}=\mathrm{pH}$ urinario delle 24 ore ; $(*)=$ rapporto tra creatininuria e idrossiprolina-telopeptide-deossipiridinolina; $(\S)=$ fosfatasi alcalina ossea e osteocalcina; $=$ =peculiarità della forma; $(\#)$ = è frequente nella pratica clinica l'associazione tra ipercalciuria ed aumento di questo parametro; $\left({ }^{\circ}\right)=$ dopo prolungato digiuno, di almeno 14 ore. 


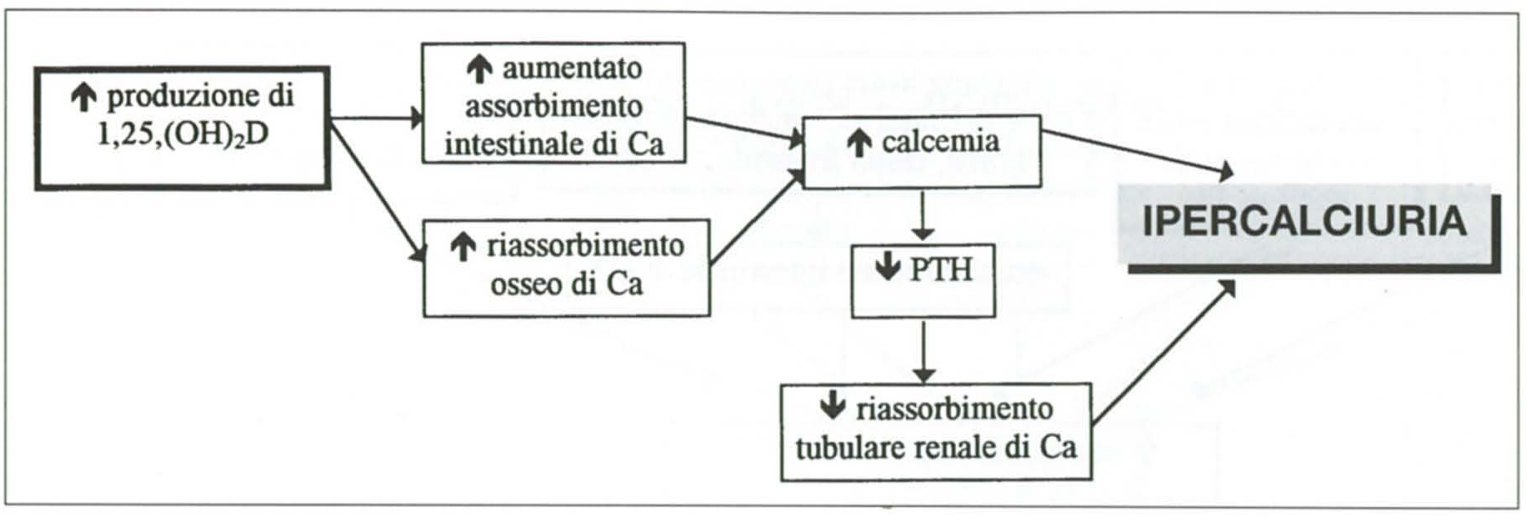

Fig. 5 - Schema patogenetico dell'Ipercalciuria da Riassorbimento osseo, secondario a primitiva iperproduzione di vit-D.

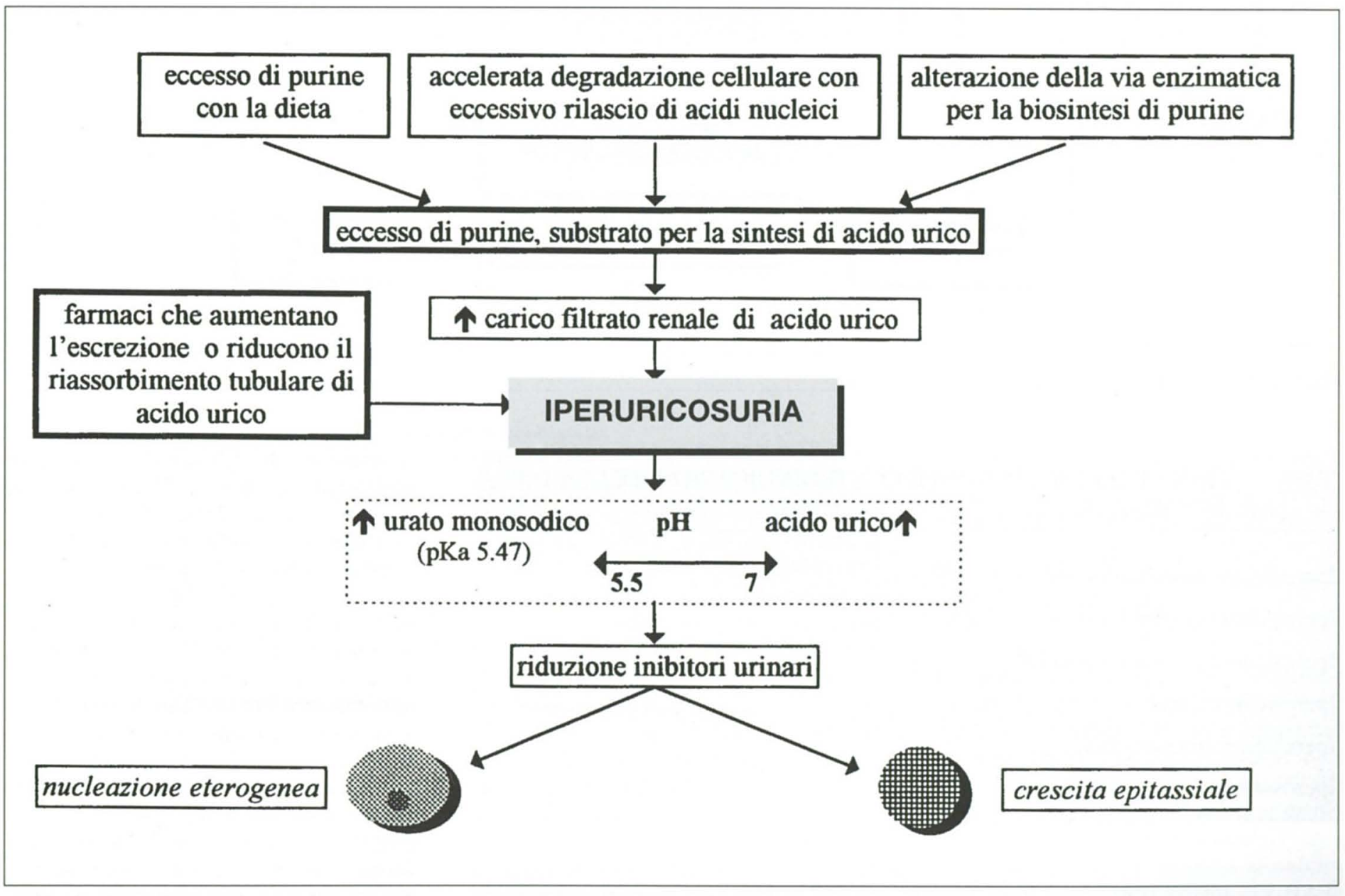

Fig. 6 - Schema patogenetico della nefrolitiasi calcica da Iperuricosuria.

tassiale, ovvero per l'interazione molecolare tra $\mathrm{OXCa}$ e acido urico/urato monosodico (18).

Urato monosodico ed acido urico sono inoltre in grado di ridurre il potere dei cosiddetti inibitori urinari (tra cui il citrato), adsorbendoli e/o riducendone la loro attività (Fig. 6), aumentando la sovrasaturazione dei sali litogeni (18).

\section{Nefrolitiasi da iperossaluria}

Solamente una minima parte $(2-10 \%)$ dell'ossalato assunto con la dieta viene assorbito dall'intestino (6). Dei 30 $\mathrm{mg} /$ die di ossalato, normalmente eliminati con le urine, circa l' $85 \%$ proviene da sintesi endogena per metabolismo del gliossilato, triptofano e glicina (di cui sono ricchi il collagene e le proteine della carne). Il restante deriva direttamente dal suo apporto esogeno con la dieta. Una volta sintetizzato od assorbito, l'ossalato non può essere ulteriormente degradato in vivo, e la sua principale via d'escrezione è quella renale. 


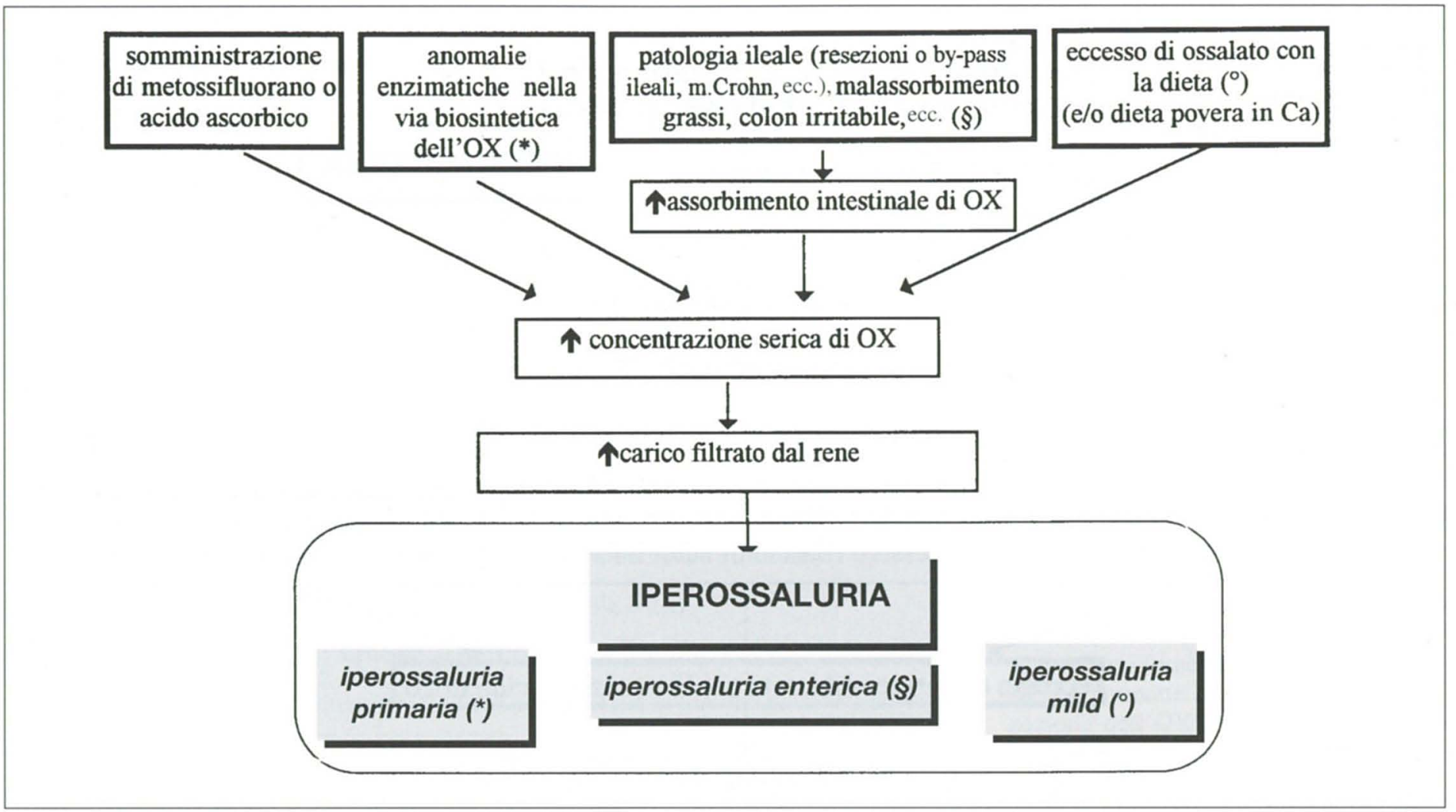

Fig. 7 - Schema patogenetico della nefrolitiasi da Iperossaluria.

\section{TAB. V - PRINCIPALI INDICAZIONI E CONTROINDICAZIONI DELLA DIETA IPOCALCICA}

Ipercalciuria assorbitiva tipo I

SI

Ipercalciuria assorbitiva tipo II

SI

Ipercalciuria assorbitiva tipo III

NO

Ipercalciuria renale

NO

Ipercalciuria riassorbitiva

NO

Iperossalurie

NO

\section{TAB.VI - PRINCIPALI FATTORI DI RISCHIO NOTI PER OSTEOPOROSI}

Familiarità per osteoporosi

Sesso femminile

Menopausa precoce

Prolungati periodi di immobilizzazione

Dieta a basso contenuto calcico

Fumo di sigaretta

Eccessivo consumo di alcolici
Il riscontro di un'escrezione urinaria giornaliera superiore ai $45 \mathrm{mg}$ di ossalato in un paziente affetto da calcolosi recidivante ossalo-calcica viene definita come Nefrolitiasi da Iperossaluria. L'iperossaluria favorisce la formazione di calcoli di OXCa per l'aumento della saturazione urinaria dei 2 sali.

Si distinguono tre principali forme di iperossaluria (Fig. 7 e Tab. VIII) (19):

- la primitiva, dovuta ad un errore congenito nella via biosintetica dell'ossalato, caratterizzata da nefrolitiasi recidivante precoce di $\mathrm{OXCa}$, e da un grave e progressivo quadro di ossalosi. In questa forma i livelli di ossaluria sono sempre elevati (>> $80 \mathrm{mg} /$ die) e nelle urine sono aumentati, a seconda dell'errore metabolico congenito, glicolato o glicerato; - l'enterica, caratterizzata anch'essa da elevati livelli di ossalato urinario (> 80 $\mathrm{mg} /$ die) ma associata a un'importante patologia intestinale, responsabile dell' $i$ perassorbimento dell'ossalato esogeno (Fig. 7). Questo avviene essenzialmente per due motivi: per l'azione irritante dei sali biliari e degli acidi grassi che aumentano la permeabilità intestinale all'OX, e per l'aumento del pool di OX-li- 


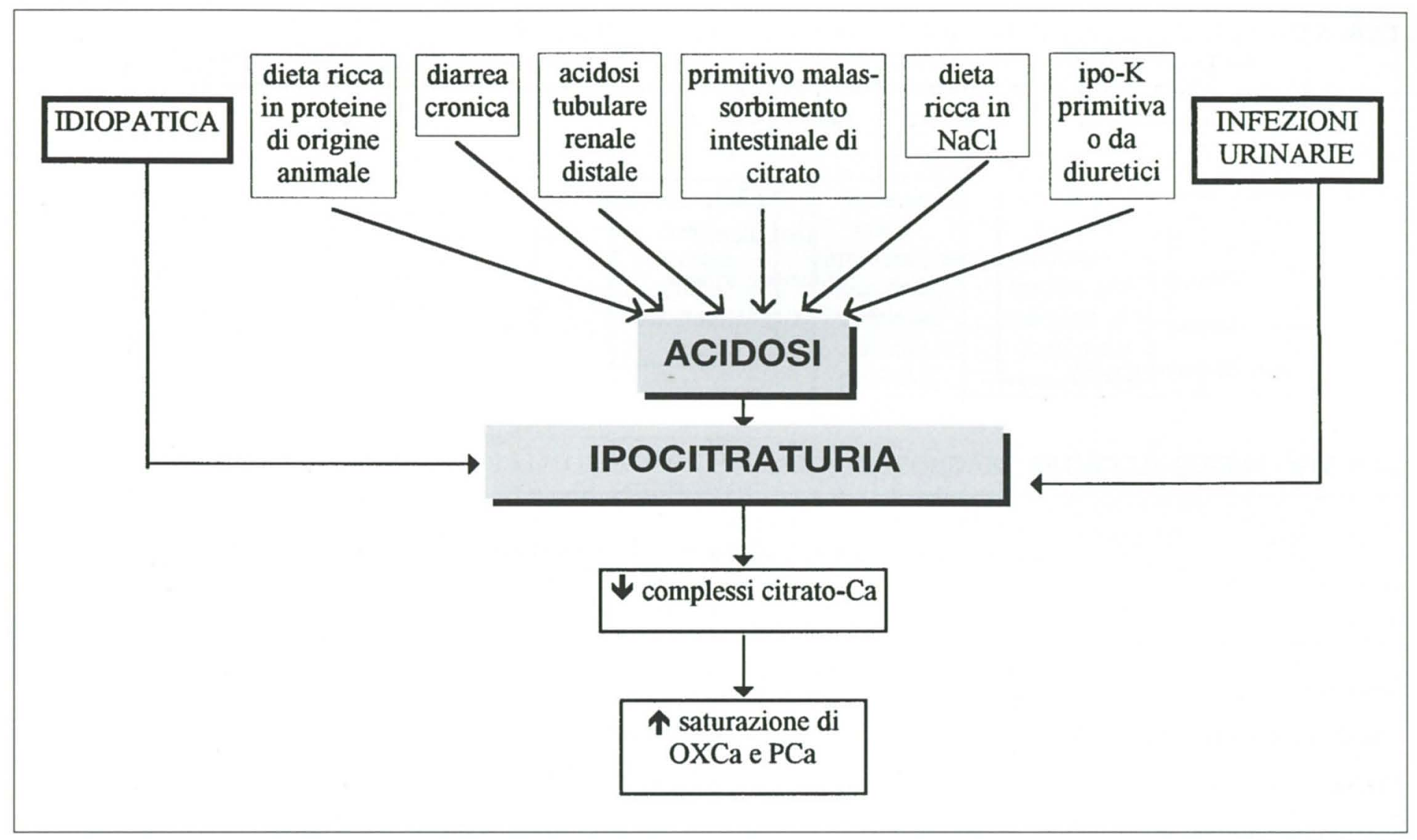

Fig. 8 - Schema eziopatogenetico della nefrolitiasi calcica da Ipocitraturia.

bero assorbibile, per sequestro di ioni divalenti (complessabili con l'OX) da parte dei saponi derivanti dal malassorbimento dei grassi (20);

- la moderata (o mild), che è la forma più frequentemente riscontrabile, in cui l'ossaluria è compresa tra 45 e 80 $\mathrm{mg} / \mathrm{die}$. La sua causa deriva da una dieta eccessivamente ricca in ossalato e spesso povera di calcio (Fig. 7). È noto infatti che il calcio è il principale chelatore intestinale (insieme a magnesio ed alti ioni divalenti) dell'ossalato ingerito (21). Nel caso specifico di calcolosi da iperossaluria-mild, la dieta ipocalcica provoca un aumento dei livelli di ossalato intestinale libero, ovvero non legato al calcio (che lo fa precipitare ed eliminare con le feci). L'ossalato libero viene così facilmente assorbito dall'apparato gastro-enterico, aumentando i suoi livelli plasmatici e quindi la sua percentuale di filtrazione renale.

Per inciso, è anche su uno di questi punti che a nostro avviso trovano origine la caduta d'interesse della classe medica e la frequente sfiducia da parte dei pa- zienti per il trattamento dietetico della nefrolitiasi. Infatti il mancato inquadramento metabolico ha fatto della cosiddetta dieta ipocalcica un indiscriminato strumento terapeutico capace in alcuni casi, paradossalmente, di peggiorare 1 'andamento della nefrolitiasi. In un paziente affetto da nefrolitiasi ossalocalcica recidivante l'affrettata prescrizione di una dieta ipocalcica rischia di essere una terapia appropriata solo nel $50 \%$ dei casi (Tab. V) (22).

Negli ultimi 15 anni ciò ha contribuito al declino della 'terapia medica della calcolosi renale'.

La dieta ipocalcica, inoltre, può anche portare ad altri effetti collaterali. Nel caso di una ipercalciuria renale o da riassorbimento osseo, ad esempio, può aggravare l'osteopenia tipica di queste forme, specie nelle persone in cui sono presenti fattori di rischio per osteoporosi (Tab. VI) $(23,24)$.

Va ricordato che l'iperossaluria mild si può associare alle forme di ipercalciuria e iperuricosuria precedentemente descritte.

\section{Nefrolitiasi da deficit di inibitori (Ipocitraturia)}

La forma più frequente di nefrolitiasi calcica da deficit degli inibitori urinari è quella da Ipocitraturia. Nelle casistiche più numerose (25) è stata riportata come 'unica anomalia metabolica' nel 5\% dei casi, e concausa delle altre forme di nefrolitiasi in circa il $50 \%$ dei casi!

Il citrato urinario, legandosi al calcio, forma dei complessi Ca-citrato altamente solubili. Così facendo, il citrato riduce la quantità libera di calcio disponibile per la formazione di calcoli di OXCa o PCa (Fig. 8) (26, 27).

L'escrezione urinaria del citrato è funzione della sua filtrazione glomerulare, riassorbimento tubulare, trasporto peritubulare (dai capillari peritubulari) e sintesi nelle cellule tubulari renali (28). Sebbene l'handling renale del citrato non sia stato ancora del tutto chiarito, si può dire che il $90 \%$ del citrato filtrato a livello 
TAB. VII - PRINCIPALI FATTORI CHE INFLUENZANO L'ESCREZIONE RENALE DI CITRATO

\begin{tabular}{cc}
\hline Aumenta l'escrezione & Riduce l'escrezione \\
\hline Alcalosi & Acidosi \\
PTH & Ipokaliemia \\
Vitamina D & Infezioni urinarie \\
Estrogeni & Androgeni \\
Ormone della crescita & \\
\hline
\end{tabular}

glomerulare viene normalmente riassorbito dai tubuli e che vi sono diversi fattori che possono aumentarlo (29) o ridurlo (26) (Tab. VII).

Tra tutti i fattori che influenzano la citraturia (Tab. VII) il più importante è certamente l'equilibrio acido-base. L'acidosi metabolica infatti, indipendentemente dalla causa che l'origina (Fig. 8), è in grado di aumentare il riassorbimento tubulare di citrato, di diminuirne l'uptake dai capillari peritubulari e la sintesi da parte delle cellule tubulari renali: ne consegue ipocitraturia $(<320 \mathrm{mg} / \mathrm{die})(26)$.

TAB. VIII - PRINCIPALI CRITERI DIAGNOSTICI DELLE NEFROLITIASI CALCICHE NON IPERCALCIURICHE

\begin{tabular}{|c|c|c|c|c|c|}
\hline & $\begin{array}{c}\text { Da Iper- } \\
\text { uricosuria }\end{array}$ & $\begin{array}{l}\text { Da Iper- } \\
\text { ossaluria }\end{array}$ & $\begin{array}{l}\text { Da Ipo- } \\
\text { citraturia }\end{array}$ & $\begin{array}{c}\mathrm{Da} \downarrow \\
\text { volume } \\
\text { urinario }\end{array}$ & $\begin{array}{c}\text { Senza } \\
\text { evidenti } \\
\text { alterazioni } \\
\text { metaboliche }\end{array}$ \\
\hline Calcemia & $\mathrm{N}$ & $\mathrm{N}$ & $\mathrm{N}$ & $\mathrm{N}$ & $\mathrm{N}$ \\
\hline Calciuria $24 \mathrm{~h}$ dieta libera & $\mathrm{N}$ & $\mathrm{N} \downarrow(\S)$ & $\mathrm{N}$ & $\mathrm{N}$ & $\mathrm{N}$ \\
\hline Calciuria $24 \mathrm{~h}$ digiuno & $\mathrm{N}$ & $\mathrm{N}$ & $\mathrm{N}$ & $\mathrm{N}$ & $\mathrm{N}$ \\
\hline Calciuria dopo carico di $\mathrm{Ca}$ & $\mathrm{N}$ & $\mathrm{N}$ & $\mathrm{N}$ & $\mathrm{N}$ & $\mathrm{N}$ \\
\hline Uricemia & $\mathrm{N} \uparrow$ & $\mathrm{N}$ & $\mathrm{N}$ & $\mathrm{N}$ & $\mathrm{N}$ \\
\hline Uricuria $24 \mathrm{~h}$ & $\uparrow$ & $\mathrm{N}$ & $\mathrm{N}$ & $\mathrm{N}$ & $\mathrm{N}$ \\
\hline Ossaluria $24 \mathrm{~h}$ & $\mathrm{~N}$ & $\uparrow\left({ }^{\circ}\right) \uparrow \uparrow(* \S)$ & $\mathrm{N}$ & $\mathrm{N}$ & $\mathrm{N}$ \\
\hline Glicolato Glicerato-u & - & $\mathrm{N} \uparrow(*)$ & - & - & - \\
\hline PTH-s & $\mathrm{N}$ & $\mathrm{N} \uparrow(\S)$ & $\mathrm{N} \uparrow(\#)$ & $\mathrm{N}$ & $\mathrm{N}$ \\
\hline Magnesiemia & $\mathrm{N}$ & $\mathrm{N} \downarrow(\S)$ & $\mathrm{N}$ & $\mathrm{N}$ & $\mathrm{N}$ \\
\hline Magnesuria 24h & $\mathrm{N}$ & $\mathrm{N} \downarrow(\S)$ & $\mathrm{N}$ & $\mathrm{N}$ & $\mathrm{N}$ \\
\hline Citraturia $24 \mathrm{~h}$ & $\mathrm{~N} \downarrow$ & $\mathrm{N} \downarrow(\S)$ & $\downarrow$ & $\mathrm{N}$ & $\mathrm{N}$ \\
\hline pH-u 24h & $\mathbf{N}$ & $\mathrm{N} \downarrow(\S)$ & $\downarrow \uparrow(\#)$ & $\mathrm{N}$ & $\mathrm{N}$ \\
\hline $\mathrm{HCO} 3-\mathrm{s}$ & $\mathrm{N}$ & $\mathrm{N} \downarrow(\S)$ & $\mathrm{N} \downarrow$ (\#) & $\mathrm{N}$ & $\mathrm{N}$ \\
\hline Kaliemia & $\mathrm{N}$ & $\mathrm{N} \downarrow(\S)$ & $\mathrm{N} \downarrow$ (\#) & $\mathrm{N}$ & $\mathrm{N}$ \\
\hline Anion gap-s & $\mathrm{N}$ & $\mathrm{N} \uparrow(\S)$ & $\mathrm{N} \uparrow(\#)$ & $\mathrm{N}$ & $\mathrm{N}$ \\
\hline Volume urinario & $\mathrm{N}$ & $\mathrm{N} \downarrow(\S)$ & $\mathrm{N}$ & $\downarrow$ & $\mathrm{N}$ \\
\hline \multirow[t]{2}{*}{ Analisi del calcolo } & $\mathrm{OXCa}$ & $\mathrm{OXCa}$ & $\mathrm{OXCa}$ & $\mathrm{OXCa}$ & $\mathrm{OXCa}$ \\
\hline & & & & $\mathrm{PCa}(\#)$ & \\
\hline
\end{tabular}

Legenda: $\mathrm{N}=$ normale; $\uparrow=$ aumento; $\downarrow=$ diminuzione; $\mathrm{PTH}-\mathrm{s}=$ paratormone serico; HCO3-s = bicarbonatemia; Anion gap-s = Na$(\mathrm{HCO} 3+\mathrm{C} 1)$ serici; $\mathrm{pH}-\mathrm{u}=\mathrm{pH}$ urinario delle 24 ore; $(*)=$ iperossaluria primitiva; $(\S)=$ iperossaluria enterica; $\left({ }^{\circ}\right)=$ iperossaluria mild; $(\#)$ = acidosi tubulare distale; $=$ pecularità della forma 


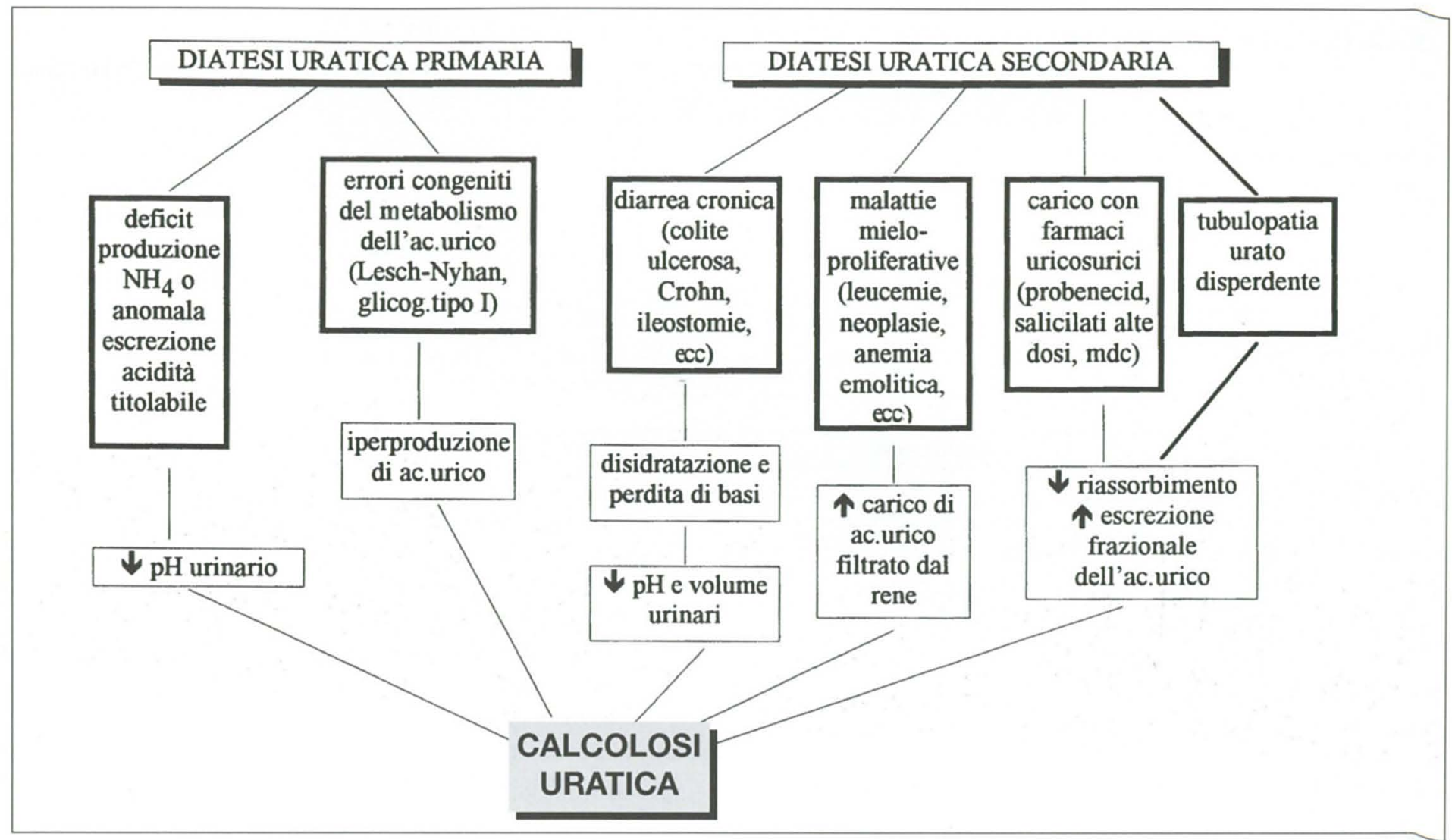

Fig. 9 - Schema patogenetico della calcolosi da acido urico.

Anche questo disordine può presentarsi in associazione con altre anomalie già descritte; i principali criteri diagnostici dell'ipocitraturia sono riportati in Tabella VIII.

\section{Nefrolitiasi da basso volume urinario $e$ senza evidenti alterazioni metaboliche}

Con un' appropriata valutazione metabolica, oltre il $95 \%$ dei pazienti affetti da nefrolitiasi recidivante trova una sua collocazione nella classificazione eziopatogenetica (Tab. II) (30). Accanto alle peculiari anomalie delle varie forme di calcolosi renale, nel $15 \%$ dei casi si associa il riscontro di un basso volume urinario (<1L/die) (Tab. VIII); questo dato non associato ad altre anomalie metaboliche incide per il $3 \%$ dei casi di nefrolitiasi (30). In meno dell' $1 \%$ dei casi non è possibile, allo stato attuale, rilevare alcuna alterazione metabolica (Tab. VIII).

\section{NEFROLITIASI NON CALCICA}

\section{Calcolosi da acido urico}

L'acido urico, prodotto terminale del metabolismo purinico, non può essere degradato dall'uomo che non possiede l'uricasi. Il rene è l'organo ove ha luogo la maggior parte della sua eliminazione, regolata dal bilancio tra sua secrezione e riassorbimento.

Il paziente affetto da calcolosi da acido urico è classicamente considerato quello con diatesi uratica (31), ovvero da una sindrome in cui la piena manifestazione si evidenzia con artropatia gottosa, iperuricemia e calcolosi uratica, mentre il quadro clinico nefrolitiasi da acido urico o mista e urine a basso $\mathrm{pH}$ viene considerato la forma iniziale o frusta. Resta il fatto che la caratteristica più comune in tale tipo di paziente è l'inusuale basso pH urinario $(<5.5)$ (Tab. IX), un pH al quale l'acido urico è assai poco solubile. Così, se da una parte non tutti i pazienti lamentano artropatia gottosa e/o iperuricemia, dall'altra l'iperuricosuria è spesso assente e neppure l'analisi chimico-fisica dei calcoli è sempre d'aiuto per la diagnosi. Infatti, accanto a calcoli composti interamente da acido urico, ve ne sono altri misti con acido urico, OXCa e/o $\mathrm{P}_{\mathrm{Ca}}$ (Tab. IX).

In Figura 9 è riportato lo schema patogenetico della sindrome. La causa del persistentemente basso $\mathrm{pH}$ urinario $(50 \%$ vs $15 \%$ dei soggetti normali [32]) non è stata ancora chiarita (33); anche la cosiddetta 'marea alcalina post-prandiale', presente nei soggetti normali, non è presente nel paziente con diatesi uratica. Non è chiaro se il difetto risieda nell'incapacità alla produzione di ammonio $\left(\mathrm{NH}_{4}\right)$ o d'escrezione dell'acidità titolabile, o ad entrambe (34).

\section{Calcolosi da cistina}

La calcolosi da cistina è la manifestazi $i_{0-}$ ne clinica di una malattia metabolica ereditaria a trasmissione autosomica recessiva: la cistinuria. La sua prevalenza $\mathrm{su}$ 
TAB. IX - PRINCIPALI CRITERI DIAGNOSTICI DELLE NEFROLITIASI NON CALCICHE

\begin{tabular}{|c|c|c|c|}
\hline & Da acido urico & Da cistina & Infetta (germi u-s) \\
\hline $\begin{array}{l}\text { Dati anamnestici } \\
\text { positivi }\end{array}$ & $\begin{array}{l}\text { Gotta/iperuricemia } \\
\text { ipertensione arteriosa } \\
\text { iperdislipidemia }\end{array}$ & Cistinuria & $\begin{array}{l}\text { Sesso femminile } \\
\text { storia di pregresse } \\
\text { infezioni urinarie }\end{array}$ \\
\hline $\mathrm{pH}$ urinario $24 \mathrm{~h}$ & $<5.5$ & $\mathrm{~N}$ & $>7-7.5$ \\
\hline $\mathrm{pH}$ urinario a digiuno & $<5$ & $\mathrm{~N}$ & $>7$ \\
\hline Iperuricosuria & Frequente & $\mathrm{N}$ & $\mathrm{N}$ \\
\hline Urocoltura & $\mathrm{N}$ & $\mathrm{N}$ & Positiva \\
\hline Leucocituria & $\mathrm{N}$ & $\mathrm{N}$ & $\mathrm{S}$ \\
\hline Cristalluria & Acido urico & Cistina & Triplofosfato \\
\hline Citraturia & $\mathrm{N} \downarrow$ & $\mathrm{N}$ & $\downarrow$ \\
\hline \multirow[t]{2}{*}{ Composizione calcoli } & Ac. urico $(50 \%)$ & Cistina & Struvite \\
\hline & $\begin{array}{c}\text { ac. urico+OXCa/PCa } \\
\text { solo } \mathrm{OXCa} / \mathrm{PCa}\end{array}$ & OXCa o ac.urico (raro) & \\
\hline Forma rx calcoli & $\begin{array}{c}\text { Varia (raramente a } \\
\text { stampo) }\end{array}$ & $\begin{array}{c}\text { Varia e policiclica, anche } \\
\text { a stampo }\end{array}$ & A stampo o varia \\
\hline Rx trasparenza o meno & Più frequente & $\begin{array}{l}\text { Non eccessivamente rx- } \\
\text { opachi }\end{array}$ & Rx-opachi \\
\hline
\end{tabular}

tutti i tipi di calcolosi varia dall' 1 al $3 \%$. I portatori omozigoti di tale anomalia congenita hanno un ridotto assorbimento intestinale di alcuni aminoacidi (cistina, lisina, ornitina e arginina) ed un' eccessiva escrezione urinaria di cistina $(>250$ $\mathrm{mg} / \mathrm{g}$ di creatinina) che normalmente è presente in minima quantità $(20 \mathrm{mg} / \mathrm{die})$ (35). La patogenesi della formazione del calcolo di cistina è dovuta all'elevata concentrazione urinaria dell'aminoacido ed alla sua bassa solubilità all'usuale $\mathrm{pH}$ urinario (massima solubilità per $\mathrm{pH}$ > 7.5). Poiché i livelli serici di cistina nei pazienti sono ridotti, si pensa che alla base della patologia nefrolitiasica ci sia un difetto 'complesso' dell'handling-tubulare dell' aminoacido: la cistina infatti, dopo essere stata filtrata a livello glomerulare, viene quasi totalmente riassorbita dal tubulo prossimale (36).

La calcolosi insorge in età precoce con un picco verso la seconda/terza decade di vita, ma può fare la sua comparsa a qualsiasi età (37). La diagnosi usualmente viene posta all'esame del sedimento urinario a fresco per riconoscimento dei tipici cristalli esagonali e confermfata dall'esame chimico-fisico dei calcoli (Tab. IX).

\section{Calcolosi infetta}

L'infezione delle vie urinarie con germi capaci di scindere l'urea (urea-splitting) è la causa principale della calcolosi infetta. Tali germi (Proteus, alcuni stafilococchi, Escherichia coli, Pseudomonas e Klebsielle) posseggono l'enzima capace di degradare l'urea in ammonio (38). Così facendo sono in grado di mantenere il $\mathrm{pH}$ urinario tanto elevato $(>7.5)$ da aumentare la dissociazione del fosfato in fosfato tribasico. Questo, precipitando con magnesio, ammonio e carbonato di calcio, forma i calcoli di struvite che sono caratteristici della calcolosi infetta. Oltre ai calcoli misti di struvite, sono caratteristici di questo tipo di calcolosi il riscontro di urocoltura positiva, leucocituria, cristalluria di triplofosfato, elevato $\mathrm{pH}$ urinario sia a digiuno che delle 24 ore e bassa citraturia (per sua degradazione batterica, Fig. 8) (Tab. IX) (38).

Lo studio metabolico per tale tipo di paziente potrebbe sembrare inutile (come del resto potrebbe apparire per la calcolosi da acido urico e cistina). È noto altresì che queste tre forme di calcolosi non calcica spesso si associano ad altri errori metabolici. Pertanto anche questi pazienti meritano sempre uno studio metabolico completo (Tab. IX).

Per la difficoltà a mantenere sterili le urine, poiché i germi 'dimorano nei calcoli' dove non sono raggiunti da efficaci concentrazioni di antibiotico, questa forma di nefrolitiasi è rimasta l'unica che vede ancora l'uso prevalente delle tecnichè chirurgiche classiche.

\section{Conclusione}

Alla luce della classificazione sopra riportata, permangono molteplici motivi per cui i pazienti affetti da nefrolitiasi non vi trovano una collocazione (Tab. $\mathrm{X})$. Tra questi riveste una particolare importanza la corretta ed accurata determinazione dei parametri biochimici (16).

I dosaggi quantitativi necessari per una valutazione metabolica della nefrolitiasi presentano difficoltà pratiche e metodologiche, che richiedono da parte dei Laboratori di Analisi una particolare attenzione, una specifica preparazione ed una adeguata strumentazione.

Poiché da tempo è noto che un corretto iter diagnostico consente di identificare 


\section{TAB. $\mathbf{X}$ - PRINCIPALI MOTIVI PER IL MANCATO INQUADRAMENTO DEI}

PAZIENTI NEFROLITIASICI

\section{Management del paziente da parte del solo medico di base}

Management del paziente solo da parte dello specialista urologo

Mancanza di un centro/specialista nefrologo per prescrivere/interpretare lo screening Impossibilità a recepire un laboratorio di analisi in grado di attuare lo screening

Mancanza di dietiste con solida esperienza di pazienti nefrolitiasici

Screening attuato in regime di ricovero

\section{TAB. XI - QUANDO RITENERE UN PAZIENTE CANDIDATO ALLA VALUTA-} ZIONE METABOLICA DELLA NEFROLITIASI

Ricorrenti episodi di nefrolitiasi calcica

Formazione di nuovi calcoli nell'ultimo anno

Aumento di dimensioni dei calcoli nell'ultimo anno

Nefrolitiasi recidivante non calcica

Necessità di frequenti procedure litotriptiche o endourologiche

Nefrolitiasi associata a fattori di rischio noi:

- maschi di media età

- presenza di stati diarroici cronici secondari a patologia intestinale

- osteoporosi

- infezioni urinarie ricorrenti

- gotta

- fattori occupazionali o ricreativi causa di disidratazione

il difetto metabolico in oltre il $95 \%$ dei casi di nefrolitiasi (9) sembra ingiustificato che esso non venga proposto nei casi di nefrolitiasi recidivante. Affermiamo ciò anche in considerazione del fatto che l'adeguato inquadramento fisiopatologico resta il prerequisito essenziale per un corretto e mirato trattamento dietetico e/o farmacologico della calcolosi renale e per la prevenzione delle sue recidive. Pertanto riteniamo che esistano dei pazienti sicuramente candidati alla valutazione metabolica, come riportato in Tabella XI.

\section{BIBLIOGRAFIA}

1. Marangella M. Metabolic evaluation of calcium nephrolithiasis. J Nephrol 1995; 8: 17984.

2. Chaussy C, Brendel W, Schmiedt E. Extracorporeally induced destruction of kidney stones by shock waves. Lancet 1980; 2 : 1265-7.

3. Resnick MI, Pak CYC. Is the metabolicwork-up of urolithiasis necessary? J Urol 1987; 137: $960-1$.

4. Marangella M, Vitale C, Petrarulo M. Valutazione metaboli- ca della nefrolitiasi calcica. Giorn Tecniche Nefrol Dial 1996; 8: 2333.

5.

Coe FL, Favus MJ, Pak CYC, Parks JH, Preminger GM. Kidney stones. Medical and surgical management. LippincottRaven publishers, Philadelphia, NW; 1996.

6. Jagger P. Renal stone disease in the 1990s: the powder keg and tinderbox theory. Current Opinion Nephrol Hypertens 1992; 1: 141-8.

7. Pak CYC, Britton F, Peterson $\mathrm{R}$, et al. Ambulatory evaluation of nephrolithiasis: classification, clinical presentation and diagnostic criteria. Am J Med 1980; 69: 19-30.

8. Pak CYC, Peters P, Hurt G, et al. Is selective therapy of recurrent nephrolithiasis possible? Am J Med 1981; 71: 615-22.

9. Lingeman JE, Preminger GM, Wilson DM. Kidney stones: identifying the causes. Patient Care 1990; 24: 31-43.

10. Minetti L, Colusso G, Surian M. Nefrolitiasi calcica. In: Minetti L, Colusso G, Surian M (Eds). Nefrolitiasi: fisiopatologia e clinica. Acta Medica Edizioni e Congressi, Roma 1985; IV: 3178.

11. Minetti L, Colusso G, Surian M. Aspetti fisicochimici della formazione dei calcoli renali. In: Minetti L, Colusso G, Surian M (Eds). Nefrolitiasi: fisiopatologia e clinica. Acta Medica Edizioni e Congressi, Roma 1985; II: 9-17.

12. Pak CYC. Physiological basis for absorbitive and renal hypercalciurias. Am J Med 1979; 237: $415 \mathrm{~F}-23 \mathrm{~F}$.

13. Brannan PG, Morawski S, Pak CYC, et al. Selective jejunal hyperabsorption of calcium in absorptive hypercalciuria. Am J Med 1979; 66: 425-8.

14. Pak CYC, Mc Guire J, Peterson R, et al. Familial absorpti- 
ve hypercalciuria in a large kindred. J Urol 1981; 126: 717-9.

15. Shen FH, Baylink DJ, Nielson RL, et al. Increased serum 1,25-dihydroxyvitamin $\mathrm{D}$ in idiopathic hypercalciuria. J Lab Clin Med 1977; 90: 955-62.

16. Borghi L, Meschi T, Novarini A. Paziente con nefrolitiasi. Profilo diagnostico di rischio litogeno e follow-up ambulatoriale. Fed Med 1993; 14: 27-34.

17. Henneman $\mathrm{PH}$, Benedict $\mathrm{PH}$, Forbes AP. Idiopathic hypercalciuria. N Engl J Med 1958; 259: 801-7.

18. Zerwekh JE, Holt K, Pak CYC. Natural urinary macromolecolar inhibitors: attenuation of inhibitory activity by urate salts. Kidney Int 1983; 23: 838-41.

19. Smith LH. Hyperoxaluric states. In: Coe FL, Favus MJ (Eds). Disorders of bone and mineral Metabolism. Raven Press, New York 1992: 707-27.

20. Dobbins JW, Binder HJ. Effects of bile salts and fatty acids on the colonic absorption of oxalate. Gastroenterology 1976; 70: 1096-100.

21. Stauffer QJ. Hyperoxaluria and intestinal disease: the role of steatorrhea and dietary calcium in regulating intestinal oxalate absorption. Digest Dis 1977; 22: 921-8.

22. Curhan GC, Willett WC, Rimm EB, Stampfer MJ. A prospective study of dietary calcium and other nutrients and the risk of symptomatic kidney stones. N Engl J Med 1993; 328: 833-8.

23. Christiansen C. Consensus developments conference: diagnosis, prophylaxis, and treatment of osteoporosis. Am J Med 1993; 94: 646-50.

24. Marangella M, Cosseddu D, Vitale C, et al. Studio dei parametri di riassorbimento osseo nelle sindromi ipercalciuriche. Pandora 1994; 6: 29-32.
25. Nicar MJ, Skurla C, Sakhaee $\mathrm{K}$, et al. Low urinary citrate excretion in nephrolithiasis. Urology $1983 ; 21: 8-14$.

26. Pak CYC. Citrate and renal calculi: new insights and future directions. Am J Kidney Dis 1991; 17: 420-5.

27. Nicar MJ, Hill K, Pak CYC. Inhibition by citrate of spontaneous precipitation of calcium oxalate, in vitro. J Bone Mineral Res 1987; 2: 215-20.

28. Baruch SB, Barich RL, Eun CK, et al. Renal metabolism of citrate. Med Clin North Am 1975; 59: 569-82.

29. Simpson DP. Regulation of renal citrate metabolism by bicarbonate ion and $\mathrm{pH}$ : observations in tissue slices and mitochondria. $\mathrm{J}$ Clin Invest 1967; 16: 225-38.

30. Levy FD, Pak CYC. Ambulatory evaluation of Nephrolithiasis: an update of a 1980 protocol. Am J Med 1995; 98: 50-9.

31. Pak CYC, Sakhaee K, Fuller C. Successfull management of uric acid nephrolithiasis with potassium citrate. Kidney Int 1986; 30: 422-8.

32. Yu TF, Gutman AB. Uric acid nephrolithiasis in gout. Predisposing factors. Ann Intern Med 1967; 67: 1133-48.

33. Hennerman PH, Wallach S, Dempsey EF. Metabolic defect responsible for uric acid stone formation. J Clin Invest 1962; 41: 537-42.

34. Gutman AB, Yu TF. A three-component system for regulation of renal excretion of uric acid in man. Trans Assoc Am Physicians 1961; 74: 353-65.

35. Halperin EC, Thier SO. Cystinuria. In: Coe FL, Brenner BM, Stein JH (Eds). Nephrolithiasis. Churchill Livingstone, New York 1980; 5 (10): 209-30.

36. Broadus A, Thier SO. Metabolic basis of renal stone disease. N Engl Med 1979; 300: 839-45.
37. Linari F, Marangella $\mathrm{M}$, Fruttero B, Bruno M. The natural history of cystinuria: a 15 year follow-up in 106 patients. In: Smith L, Robertson W, Finlayson B (Eds). Urolithiasis, clinical and basic research. Plenum Press, New York 1981: 145-54.

38. Griffith DP. Struvite stones. Kidney Int 1978; 13: 372-82. 